\title{
Multiple splicing variants of Naf1/ABIN-1 transcripts and their alterations in hematopoietic tumors
}

\author{
YASUHIRO SHIOTE ${ }^{1,2}$, MAMORU OUCHIDA ${ }^{1}$, YOSHIMI JITSUMORI ${ }^{1}$, YOICHIRO OGAMA ${ }^{3}$, \\ YOSHINOBU MATSUO ${ }^{4}$, FUMIHIKO ISHIMARU ${ }^{2}$, MITSUNE TANIMOTO ${ }^{2}$ and KENJI SHIMIZU ${ }^{1}$ \\ ${ }^{1}$ Departments of Molecular Genetics, ${ }^{2}$ Internal Medicine II, Okayama University Graduate School of Medicine, \\ Dentistry and Pharmaceutical Sciences, Shikata-cho 2-5-1, Okayama 700-8558, ${ }^{3}$ Department of Nephrology, \\ Higashiosaka City General Hospital, Nishiiwata 3-4-5, Higashiosaka 578-8588, ${ }^{4}$ Fujisaki Cell Center, \\ Hayashibara Biochemical Laboratories Inc., Fujisaki 675-1, Okayama 702-8006, Japan
}

Received May 19, 2006; Accepted July 13, 2006

\begin{abstract}
Naf1 (Nef-associated factor 1)/TNIP1/ABIN-1 (A20-binding inhibitor of $N F-\kappa B$ activation) is a cellular protein that interacts and cooperates with the NFKB inhibiting protein A20. It is reported that Naf1 attenuates epidermal growth factor (EGF)/extracellular-signal-regulated kinase2 (ERK2) nuclear signaling. Naf1 also binds to Nef, which plays a key role in acquired immunodeficiency syndrome pathogenesis and HIV-1 virus replication. Naf1 mRNA consists of 18 exons and multiple splice variants have been reported; two isoforms for exon 1, deletion of exon 2 and isoforms $\alpha$ and $\beta$ for exon 18 . Using specimens from 29 acute myeloid leukemia (AML) patients, we detected a high frequency of allelic loss on DNA at STS marker D5S2014 near the Nafl gene. We therefore performed mutation and expression analyses using leukemia-lymphoma lines and 6 pairs of clinical AML samples. There was no mutation in the Nafl coding region of any sample. As a result of expression analysis, we identified novel splice variants of the Nafl gene; deletion of exon 16 ( $\mathrm{Naf} 1 \alpha 2$, Naf1 B2), deletion of exon 16 with an insertion (Naf1 $\alpha 3$, Naf1 B3) and deletion of exons 16 and 17 (Naf1 $\alpha 4)$. Naf1 $\alpha 3$ and $B 3$ showed premature termination. In peripheral blood mononucleocytes (PBMNCs) from healthy adults, almost no expression of full-length Naf1 $\left(\mathrm{Naf} 1^{\mathrm{FL}}\right), \mathrm{Naf} 1 \alpha 3$ and $\mathrm{B3}$ were observed. In contrast, their expression was clear in AML blasts and in the majority of leukemia-lymphoma lines investigated. Naf1 $\alpha 2$ was widely expressed in PBMNCs from healthy adults, AML blasts and cell lines, suggesting it is the main transcript of the Nafl gene. Luciferase assay revealed that Naf1 $\alpha 2$ had equal NF-кB
\end{abstract}

Correspondence to: Dr Kenji Shimizu, Department of Molecular Genetics, Okayama University Graduate School of Medicine, Dentistry and Pharmaceutical Sciences, Shikata-cho 2-5-1, Okayama 700-8558, Japan

E-mail: shimke47@md.okayama-u.ac.jp

Key words: alternative splice variant, loss of heterozygosity, acute myeloid leukemia, nuclear factor $\kappa \mathrm{B}, \mathrm{Naf} 1 / \mathrm{ABIN}-1 / \mathrm{TNIP1}$ inhibitory effect to that of $\mathrm{Naf} 1^{\mathrm{FL}}$, while Naf1 $\alpha 4$ was less effective. In clinical AML patients, the expression of Naf1 $\alpha 3$ was much higher at diagnosis than on remission after chemotherapy, suggesting the possible dominant negative effect of Naf1 $\alpha 3$.

\section{Introduction}

Acute myeloid leukemia (AML) is characterized by an increase in the number of myeloid cells in the bone marrow and an arrest in their maturation, frequently resulting in hematopoietic insufficiency (granulocytopenia, thrombocytopenia, or anemia), with or without leukocytosis (1). It is a heterogeneous disease without a unifying genetic lesion.

Cellular transformation, as well as leukemogenesis, is considered to be a multistep process. The creation of fusion genes by chromosomal translocations, i.e. AML/ETO, $P M L / R A R A$, and $C B F A / M Y H 11$, has been postulated to be a primary event in leukemogenesis (2). However, the products of these fusion genes appear insufficient by themselves to cause AML (3-9), and it is considered that cooperating molecular genetic abnormalities are also required (1). In solid tumors, loss of heterozygosity ( $\mathrm{LOH}$ ) leading to loss of function of tumor suppressor genes (TSGs) plays a critical role in neoplastic progression and may have prognostic importance $(10,11)$. However, the role of $\mathrm{LOH}$ in the pathogenesis of leukemias has not been fully established. Current evidence suggests that TSGs may also be important in the pathogenesis of AML, for example, down-regulation of $p 27$ (Kipl) and p15/INK4b $(12,13)$.

Partial deletion within the long arm of chromosome 5 is one of the nonrandom abnormalities observed in primaryand therapy-induced myelodysplastic syndrome (MDS) and AML. These chromosomal losses are most often observed at 5q15-33 in adult de novo AML patients (14).

The chromosome 5 deletions are seen in $<10 \%$ of de novo AML cases, but in secondary AML/MDS their prevalence reaches $\sim 50 \%$ and they are frequently associated with additional chromosomal abnormalities (15). The consistent occurrence of deletions in AML/MDS suggests that a TSG, which is important in hematological transformation, resides on $5 q(16)$. 
Naf1 (Nef-associated factor-1), alternatively called as TNIP1 (TNFAIP3-interacting protein 1) or ABIN-1 (A20binding inhibitor of $\mathrm{NF}-\kappa \mathrm{B}$ activation) is a cellular protein expressed in various human tissues and cell lines. It is found independently as one of the interacting proteins with human immunodeficiency virus type-1 (HIV-1) viral protein Nef (17) or cytoplasmic zing finger protein A20 (18). A20 inhibits nuclear factor $\kappa \mathrm{B}(\mathrm{NF}-\kappa \mathrm{B})$ activity and tumor necrosis factor (TNF)-mediated programmed cell death $(19,20)$. A20deficient mice are hypersensitive to TNF, and suffer from severe inflammation correlated with enhanced NF- $\mathrm{BB}$ activity (20). A20-binding protein Naf1/ABIN-1 also has the potential to inhibit TNF-induced activation of NF- $\kappa$ B upon overexpression, suggesting that Naf1/ABIN-1 mediates the NF- $\kappa$ B inhibitory function of A20 (18). Naf1/ABIN-1 can also bind the MAP kinase ERK2 and cause its retention in the cytoplasm, leading to a reduction of ERK2 nuclear signaling which is important for cell growth, differentiation, and cell death (21).

Naf1/ABIN-1 protein has four putative leucine zippers and four predicted regions of coiled coil structures. The gene encoding Naf1/ABIN-1, which consists of 18 exons, is located on human chromosome 5q32-33.1 (17). Naf1 mRNA is ubiquitously expressed in several human tissues, with strong expression in peripheral blood lymphocytes, the spleen, and skeletal muscle. Naf1/ABIN-1 is also detected in various human hematopoietic cell lines, such as Jurkat, Molt-4, H-9 and HL60 $(17,22)$.

We detected a high LOH frequency at STS marker D5S2014 on 5q32. Searching for candidate TSGs for AML, we investigated the Naf1/ABIN-1 gene which is located near the marker. We identified novel Naf1/ABIN-1 variants and investigated the expression of these variants in leukemialymphoma cell lines and in specimens from AML patients at diagnosis and on their remission after chemotherapy.

\section{Materials and methods}

LOH analysis. Primers for amplification of microsatellite markers, D5S414, D5S500, D5S658, D5S2017, D5S436, D5S2090, D5S2014 and D5S1978 are available through the internet genome database (http://gdbwww.gdb.org/). Primers for markers MS1, MS3, MS4, MS6, MS8 and GRAF-MS1 were designed in our laboratory as follows; MS1: forward, 5'-GAA ATG CAG ACA CAC CTC AT, reverse, 5'-TTC TCT GTA AGG GTT TGG ATT; MS3: forward, 5'-AGG CTG ATG CAC CAC CCC C, reverse, 5'-ATT ACA GAC CAA GAA CAC CCT G; MS4: forward, 5'-GCT TTG GGC CGT TTC ACT TCA, reverse, 5'-TTC CCT TTT GGT CTC TCT TGT G; MS6: forward, 5'-AGG TCT TCT ATT ACA GGC CCC, reverse, 5'-GAG GTC GTT TTG TTC CTG GC; MS8: forward, 5'-CAC CCT CCT GCC CCT CTT T, reverse, 5'-TTT TAA GTG AGG TGT GTA TGT G; GRAF-MS1: forward, 5'-GAC TCC TGG CAT TGT ACT AGC, reverse, 5'-GAA ACG AGA AAA ATG CTA TGG AG. Forward primers were labeled with iodoacetamidofluorescein at the 5'end and used for PCR. PCR was carried out in $20 \mu \mathrm{l}$ of reaction mixture with $20 \mathrm{pmol}$ of each primer, $100 \mathrm{ng}$ of genomic DNA, IX PCR buffer, 200 pM of each deoxynucleotide triphosphate, and 0.5 unit of Taq DNA polymerase (Takara,
Kyoto, Japan). Initial denaturation at $94^{\circ} \mathrm{C}$ for 3 min was followed by 35 cycles of a denaturation step at $94^{\circ} \mathrm{C}$ for $30 \mathrm{sec}$, an annealing step at $62^{\circ} \mathrm{C}$ (D5S500, D5S2090), $60^{\circ} \mathrm{C}$ (D5S414, D5S658, D5S2017, D5S2014, D5S1978, MS3, MS4, MS6 and MS8) or $58^{\circ} \mathrm{C}$ (D5S436 and MS1) for $30 \mathrm{sec}$, and an extension step at $72^{\circ} \mathrm{C}$ for $1 \mathrm{~min}$. A final extension step at $72^{\circ} \mathrm{C}$ for $7 \mathrm{~min}$ was added. PCR products were analyzed on a PRISM 3100 Analyzer with GeneScan Analysis software version 3.7 (Applied Biosystems). LOH was scored if one heterozygous allele showed at least $25 \%$ reduced intensity in tumor DNA compared to the corresponding normal DNA, which was validated by calibration with reconstituted experiments.

Cell lines and primary tumors. Human lymphoid and myeloid leukemia cell lines were supplied by either the Health Science Research Resources Bank (Osaka, Japan), Hayashibara Inc. (Okayama, Japan), or the German Collection of Microorganisms and Cell Cultures (Germany), and cultured in RPMI-1640 supplemented with $10 \%$ fetal bovine serum. Human embryonic kidney HEK293T cells were grown in Dulbecco's modified Eagle's medium (DMEM) supplemented with $10 \%$ bovine serum and antibiotics. Peripheral blood (PB) and bone marrow (BM) were obtained from AML patients and healthy volunteers at the Department of Internal Medicine II after acquisition of written informed consent. Peripheral blood mononuclear cells were isolated from these PB or BM samples by FicollHypaque density centrifugation (Amersham Bioscience, Piscataway, NJ, USA) according to the manufacturer's instructions. Genomic DNAs were isolated by sodium dodecyl sulfate (SDS)/proteinase K treatment, phenol/chloroform extraction and ethanol precipitation. Total RNA was extracted using a modified acid guanidinium phenol chloroform method (Isogen, Nippon Gene, Tokyo, Japan) following the manufacturer's instructions.

$R T$-PCR and cDNA sequencing. cDNA was synthesized using the ReverTraAce kit (Toyobo, Tokyo, Japan) with random primers and oligo dT according to the manufacturer's instructions. The whole coding region of Naf1-cDNA was amplified into three overlapping fragments by RT-PCR with three pairs of the following primers: sense 1c, 5'-GTG TCC CCA AGC TCC CCG T and antisense, 5'-AGC CTG ATC CCG CTG TCC CA for Naf1-A (769 ntd, exons 1-7); sense, 5'-AGG TGC ACA AGA ATG AGC AGC and antisense, 5'TGC TGT TGG TGG AGA TGA TGG for Naf1-B (728 ntd, exons 6-13); and sense, 5'-ACC CGA CAG CGT GAG TAC CA and antisense, 5'-GGA GCC AAA TGA CAC AAT CTG $\mathrm{G}$ for Naf1-C $\alpha$ (720 ntd, exons 12-18) or antisense, 5'-TGT CGA CAC CGT GCA AGT GGC TTC TAG for Naf1-C B (791 ntd, exons 12-18). In order to detect splice variants in the $\mathrm{N}$-terminal region, primers for exon $1 \mathrm{a}$ and exon $1 \mathrm{~b}$ were designed: sense-primer for exon 1a, GAG GAG CCT TCA TAG GGA CAG; sense-primer for exon 1b, AGG AGC CCT AGG AGT GCT AC.

Cloning of Nafl cDNA variants. Total RNA (2 $\mu \mathrm{g})$ of NALL-1 was used in the reverse transcription (RT) reaction. Naf1 cDNAs were amplified by PCR with sense primer for 
A

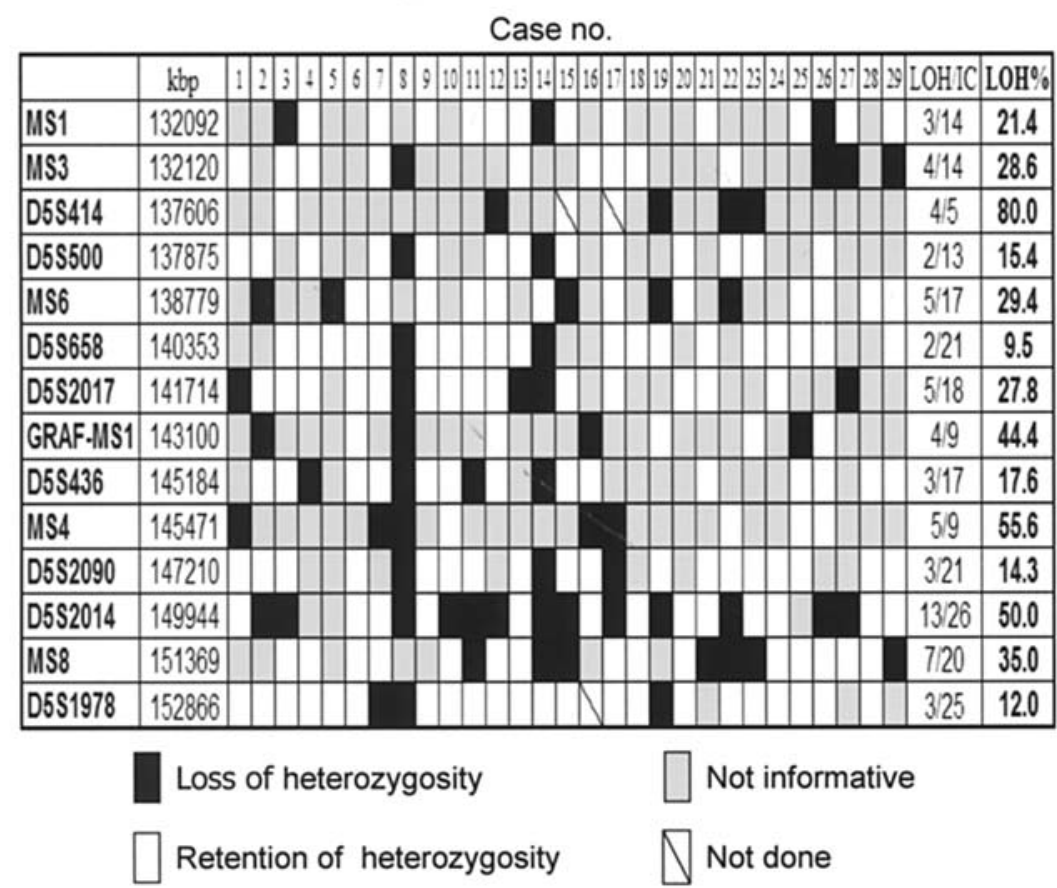

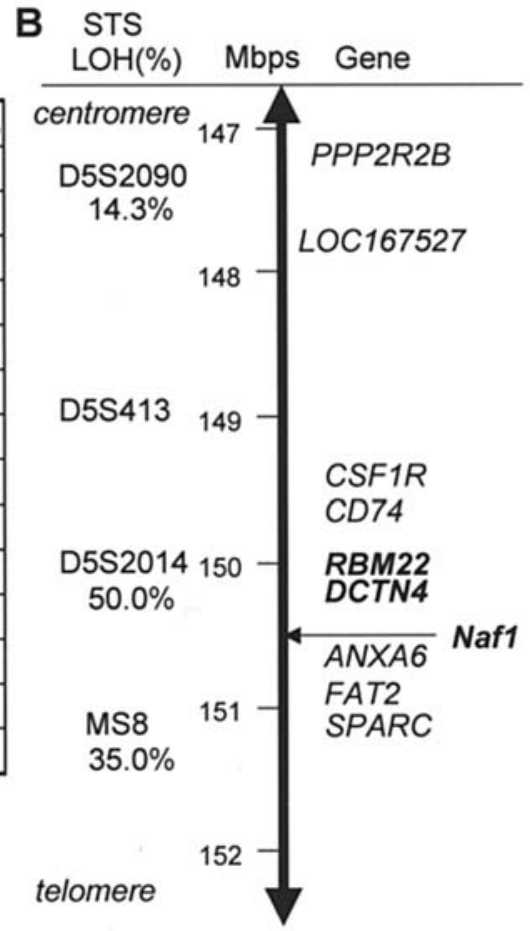

Figure 1. LOH analysis on chromosome 5q31-35. (A) Schematic representation of LOH distribution. Case numbers are shown at the top. Microsatellite markers used are shown to the left. Filled box, LOH; open box, retention of heterozygosity; shaded box, not informative (homozygous); box with slash, not completed. (B) STS map of the 5q31-32 region. The scale indicate distance from the 5pter. STS markers are shown on the right and candidate genes are shown on the right of the scale. LOH percentage on each STS marker are shown; MS8 is our original marker.

fragment $A$ and antisense primers for fragment $C(\alpha$ and $\beta)$. The PCR products were inserted into pBluescript vector at EcoRV site, and sequenced. The cDNAs were digested with SpeI and SalI, and subcloned into the pCI-bsr vector at the NheI and SalI sites to produce pCI-bsr-Naf1 $\alpha$, pCI-bsr-Naf1 $\alpha 2$, and pCI-bsr-Naf1 $\alpha 4$.

Cell culture and isolation of cell clones expressing Nafla. Human embryonic kidney HEK293 cells were cultured in DMEM containing $10 \%$ fetal bovine serum (FBS) at $37^{\circ} \mathrm{C}$ in an atmosphere of $5 \% \mathrm{CO}_{2}$. The cells were transfected with $2 \mu \mathrm{g}$ of pCI-bsr-Naf1 $\alpha$, pCI-bsr-Naf1 $\alpha 2$, pCI-bsr-Naf1 $\alpha 4$, or the empty vectors by a calcium phosphate precipitation method. After $48 \mathrm{~h}$ of transfection, the cells were cultured for two weeks in the presence of $2 \mu \mathrm{g} / \mathrm{ml}$ Blasticidin $\mathrm{S}$ (Funakoshi, Tokyo) by replacing with fresh medium containing Blasticidin $\mathrm{S}$ every 5 days. The colonies in the dish were mixed and used for reporter assay as described below.

$N F-\kappa B$ reporter gene assay. The pre-transfected HEK293T cells were grown in 48 -well plates. NF-кB luciferase reporter plasmid pNF-кB-Luc (Stratagene, La Jolla, CA) (0.05 $\mu \mathrm{g})$ was cotransfected with $0.025 \mu \mathrm{g}$ of an internal control Renilla luciferase plasmid pRL-TK (Promega, Madison, WI), $0.075 \mu \mathrm{g}$ of pFC-MEKK (Stratagene) or empty plasmid into the HEK293 cells using the calcium phosphate method. The cells were harvested $48 \mathrm{~h}$ after transfection. The activity of firefly and Renilla luciferases were measured using the DualLuciferase Reporter assay kit (Promega) and normalized for the variation in transfection efficiency. These assays were repeated twice in duplicate.

\section{Results}

Loss of heterozygosity analysis of chromosome 5q31-35 region. To examine allelic loss in the 5q31-35 region, we selected 14 microsatellite markers for LOH analysis (Fig. 1). We analyzed 29 pairs of matched AML blasts at diagnosis and peripheral blood mononucleocytes (PBMNCs) on remission. Primers MS1, 3, 4, 6 and 8 were designed as described. LOH on markers D5S414, GRAF-MS1and MS4 showed high frequency, but they had few informative cases. Excluding these markers, the microsatellite analysis showed high LOH at markers MS3, MS6, D5S2014 and MS8. As candidate tumor suppressors, we selected target genes around D5S2014, where the highest LOH was observed (13 out of 26 informative cases, 50\%). These were RNA binding motif protein 22 (RBM22), dynactin 4 (DCTN4) and Nafl genes located on 5q32-33.1. Mutation analyses of the RBM22 and DCTN4 gene were performed with blast cells from 29 AML patients, but no mutation was detected. Therefore, we next investigated the Nafl gene, located about $450 \mathrm{kbp}$ telomeric to the STS marker D5S2014.

Mutation analysis of the Nafl gene. We examined the entire coding region of Nafl on three different overlapping fragments (Naf1-A, Naf1-B and Naf1-C) sized 720-769 bp at the mRNA level by RT-PCR and direct sequencing in 16 leukemia-lymphoma cell lines as well as specimens from 29 
A

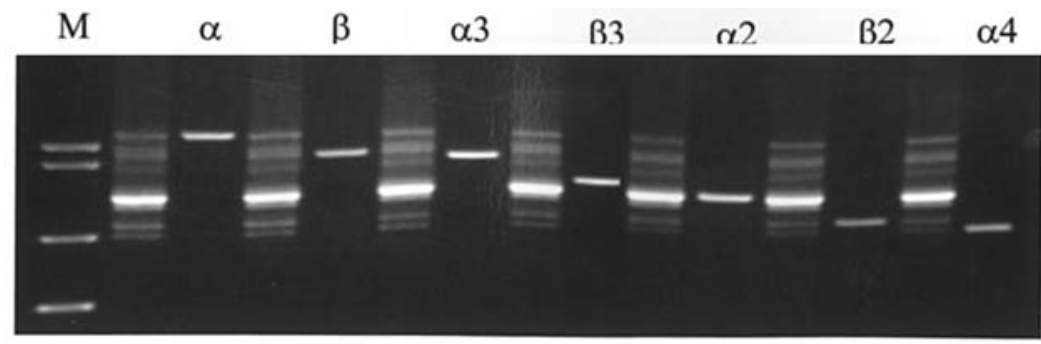

B
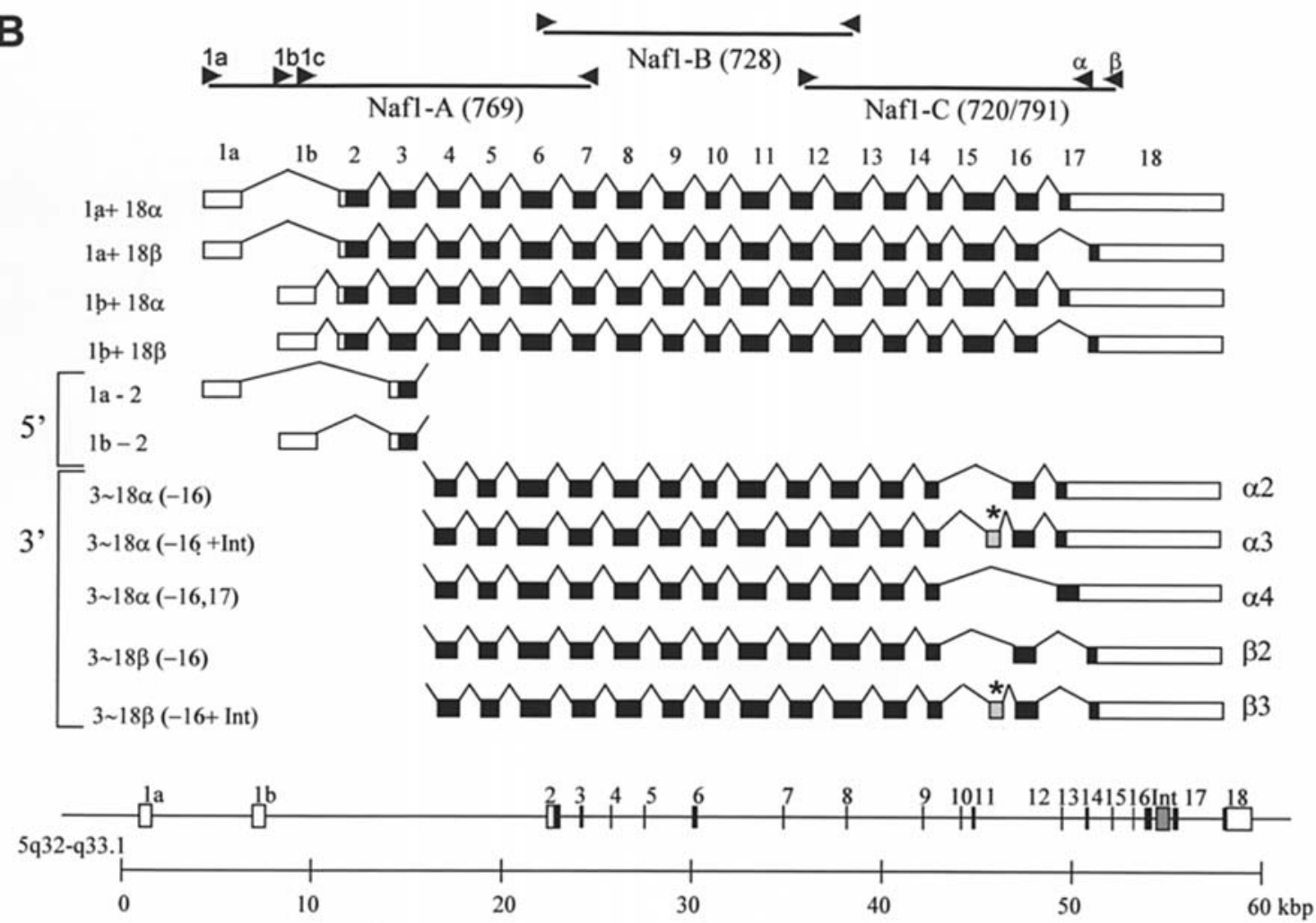

Figure 2. (A) Naf1 splice variants obtained by cloning method. Naf1 $\alpha, \beta$ and the novel five splice variants are paired by the RT-PCR product of the same sample from an AML patient (number 6) at diagnosis. M, size marker. (B) Schematic illustration of Naf1 mRNA alternative splicing isoforms. RT-PCRfragments are shown at the top. Arrow heads indicate the sense and antisense primers. RT-PCR product size for each fragment are shown. Naf1-A fragment size shown is that of RT-PCR product with primers $1 \mathrm{c}$ and antisense primer for Naf1-A. Closed boxes show the region that was spliced into the mRNAs, and open boxes show the untranslated regions. Shaded boxes show the intron insertion. * Premature termination site.

AML patients. However, no mutation was detected in any of the samples except some synonymous single nucleotide polymorphisms.

Identification of Nafl splice variants. Since a somatic mutation of the Nafl gene was not detected in the tumor cells, we looked for other possible inactivation mechanisms. We examined the expression level of Naf1 mRNA in 16 hematopoietic cell lines and PBMNCs from healthy volunteers. PCR primers were designed to encompass the exon/intron junctions on cDNA in order to eliminate the potential contamination of genomic DNA. We detected alternative splice bands in Naf1-A and Naf1-C (GenBank accession numbers AB252970-AB252980). RT-PCR of the Naf1-A showed a shorter alternative band. We isolated the band and sequenced. The sequence analysis revealed that this alternative band represented a deletion of exon 2, producing 172 bp shorter transcripts. Since exon 1 is non-translated exon, deletion of exon 2 leads to starting of the translation from the first methionin in exon 3 of the Nafl gene, producing proteins which lack the $\mathrm{N}$-terminal part of 53 amino acids. This alternative splicing has been reported by other groups (23), together with two different types of exon 1 , i.e. exon $1 \mathrm{a}$ and exon $1 \mathrm{~b}$ (23), produced by alternative promoter (Fig. 2). Exon 1a is located upstream of exon 1b, and both are non-translated exons. We detected a loss of exon 2 for both exon 1a and exon $1 \mathrm{~b}$ (Fig. 2B). On the other hand, in the $\mathrm{C}$-terminal region of $\mathrm{Naf} 1$, it has been reported that there are $\alpha$ and $\beta$ types of variants according to the alternative splicing of exon 18 (21). By RT-PCR analysis for Naf1 exon 12 to exon 18 (Naf1-C), however, we observed multiple splice variants in addition to the isoforms $\alpha$ and $B$. We next tried to identify these novel Naf1 variants.

Cloning of Naf1 variants. Our results demonstrated that there are additional splice variants for Naf1-C. In order to isolate these undefined multiple splicing bands we performed cloning (Fig. 2B). As a result, we determined five new Naf1 

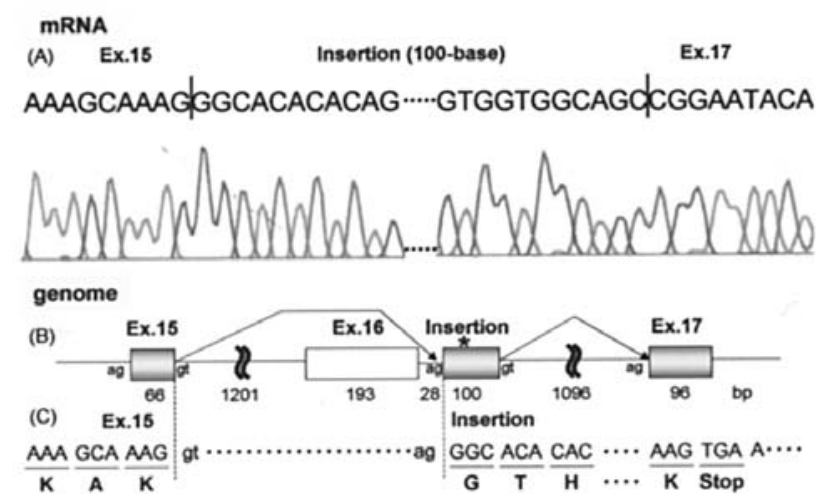

Figure 3. A splicing variant of the Naf1 gene with deletion of exon 16 and a 100 ntd insertion (Naf1 $\alpha 3$ and B3). (A) Sequence data of Naf1 $\alpha 3$ or B3. Sequence on the left shows exon 15-insertion boundary. Sequence on the right shows insertion-exon 17 boundary. Bars indicate the boundaries. (B) Schematic illustration of Naf1 $\alpha 3$ or 33 . Shaded boxes show the region that was spliced into the mRNAs, and open boxes show the exon that was spliced out. Arrows show the alternative splicing pattern. Numbers below each boxes or lines show the size (base pair) of each exon or intron. *Premature termination site. (C) Nucleotide and amino acid sequences of Naf1 $\alpha 3$ or B3. Upper-case letters show exons, and lower-case letters show splicing junctions in introns. Amino acids are shown according to triplet nucleotides with underlining.

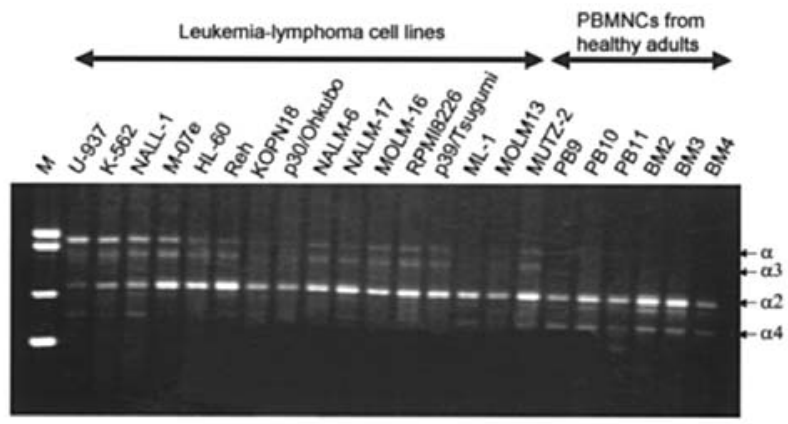

Figure 4. Expression analysis of the Naf1 gene in leukemia-lymphoma cell lines and human peripheral blood mononucleocytes (PBMNCs). RNAs were isolated from sixteen leukemia-lymphoma cell lines and PBMNCs from healthy adults. RT-PCR was performed with specific primers for Naf1 $\alpha$-C. Numbers on top of the right six lanes indicate sample number. The sources of these six samples are shown. PB, peripheral blood; BM, bone marrow; $\mathrm{M}$, size marker.

variants; variants that lack exon 16 in both $\alpha$ and $\beta$ types of Naf1 (Naf1 $\alpha 2$ and Naf1 32 respectively); variants that lack exon 16 and has 100 bp insertion between exons 15 and 17, also for $\alpha$ and $B$ type of Naf1 (Naf1 $\alpha 3$ and Naf1 B3); and a variant that lack exons 16 and 17 , which we could clone only $\alpha$ type (Naf1 $\alpha 4)$ (Fig. 2A and B). The insertions in Naf1 $\alpha 3$ and Naf1 B3 had the sequence of a part of intron 16 . We found that the deletion of exon 16 was in-frame, so Naf1 $\alpha 2$ or $\mathrm{B} 2$ transcripts has identical amino acids to $\mathrm{Naf} 1^{\mathrm{FL}}$ except that they lack 64 amino acids corresponding to exon 16 . On the other hand, in Naf1 $\alpha 3$ and $\beta 3$, which have $100 \mathrm{bp}$ insertions, premature termination occurred at the 49th ntd within the insertion (Figs. 2A and 3).

Expression analysis of Naf1 mRNA in hematopoietic cell lines. In order to analyze mRNA expression of the Nafl gene

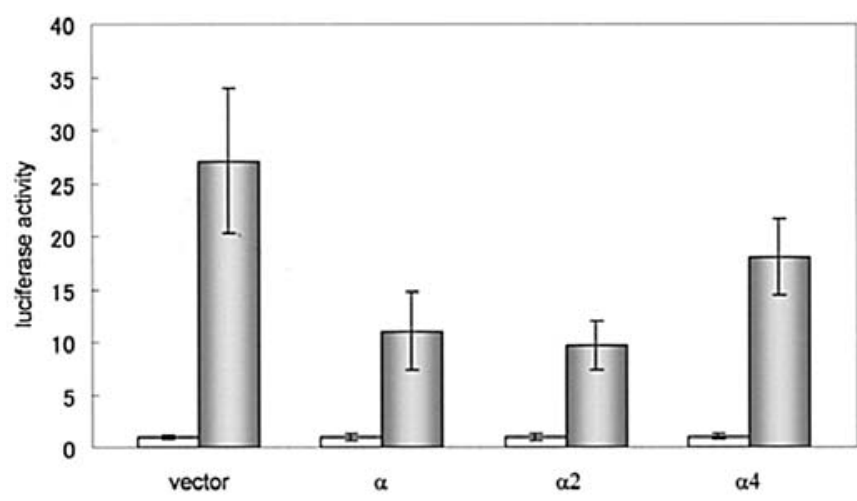

Figure 5. The effect of Naf1 variants on MEKK-induced NF- $\mathrm{BB}$ activation. HEK293 cells were transiently cotransfected with the indicated Naf1expression plasmids, an $\mathrm{NF}-\mathrm{\kappa B}$ dependent luciferase reporter plasmid, and MEKK-expression plasmid (closed bars) or empty plasmid (open bars). The effect of Naf1 $1^{\mathrm{FL}}$, Naf1 $\alpha 2$ and Naf1 $\alpha 4$ on NF-KB dependent luciferase activity was measured. The luciferase activities were expressed as the mean \pm SD from four independent experiments.

in hematopoietic cell lines, 16 leukemia-lymphoma cell lines were prepared. PBMNCs were obtained from BM or PB of healthy volunteers. Total RNA was extracted from these cell lines or PBMNCs and RT-PCR was performed with specific primers for the Naf1 $\alpha-\mathrm{C}$. We observed Naf1 $\alpha^{\mathrm{FL}}$ expression only among cell lines (at least 12 out of 16) and not in PBMNCs from healthy volunteers (Fig. 4). On the other hand, Naf1 $\alpha 2$ showed ubiquitous expression among cell lines and PBMNCs from healthy volunteers. Moreover, it showed the highest expression in most of the cell lines (other than U-937, K-562 and NALL-1, which show the highest expression in Naf1 $\alpha$ ) and healthy volunteers (Fig. 4). These results show that Naf1 $\alpha 2$, not Naf1 $\alpha$, is the main transcript of the Naf1 gene in normal PBMNCs of healthy adults. We also observed that Naf1 $\alpha 4$ expression was clear only in PBMNCs from healthy volunteers while its expression in cell lines was very low or even could not be detected (Fig. 4).

$N F-\kappa B$ inhibitory effect of Naf1 isoforms. In order to compare the function of these Naf1 isoform proteins, human embryonic kidney HEK293 cells were transfected with the Naf1 ${ }^{\mathrm{FL}}$, Naf1 $\alpha 2$, Naf1 $\alpha 4$ and empty expression vectors, selected with Blasticidin S for two weeks, and collected by trypsinization. Then, each group of transfected HEK293 cells was transfected with cDNA expression vector for MEKK, an

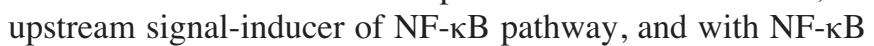
luciferase reporter plasmid bearing the $\mathrm{NF}-\kappa \mathrm{B}$ binding DNA sequences, and the luciferase activity was measured $48 \mathrm{~h}$ after transfection. As a control, the vector-transfected HEK293 cells showed a 27-fold increase through NF-кB activated by the MEKK signal (Fig. 5). But when pretransfected with $\mathrm{Naf}^{\mathrm{FL}}$ expression plasmid, NF- $\mathrm{\kappa B}$ activity increased only to 11 -fold, which is $40.8 \%$ of the control $(\mathrm{p}=0.009)$. When pre-transfected with Naf1 $\alpha 2$ expression plasmid, NF- $\kappa \mathrm{B}$ activity increased 9.6 -fold (35\% of the control; $\mathrm{p}=0.003)$, and when pre-transfected with Naf1 $\alpha 4$ expression plasmid, this increased 17 -fold $(66.4 \%$ of the control; $\mathrm{p}=0.064)$. These data indicate that Naf1 $\alpha 2$ shows 


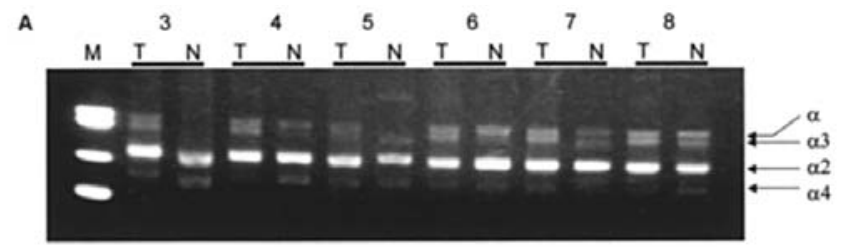

B

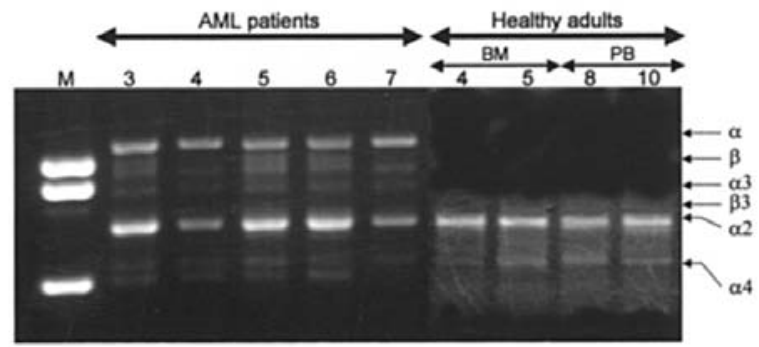

Figure 6. Naf1 expression in acute myelogenous leukemia (AML) patients. (A) RT-PCR products from AML clinical samples. Upper row indicates patient number. Tumor $(\mathrm{T})$ samples were obtained form bone marrow or peripheral blood from AML patients at diagnosis. Percentages of leukemia blasts were $>74 \%$. Normal (N) samples were obtained from PBMNCs of corresponding patients on their remission after chemotherapy. Percentage of leukemia blasts were $<1 \%$. (B) RT-PCR product of AML blasts were compared to PBMNCs from healthy adults. Lane 2-6 are from AML patients at diagnosis. Lane 7-10 are from PBMNCs from healthy adults. Numbers above each lane indicate patient number. PCR primers were selected so that ß-type variants would be included. PB, peripheral blood; BM, bone marrow.

almost equal or even higher NF-кB inhibitory effect than that of $\mathrm{Naf}^{\mathrm{FL}}$. Naf1 $\alpha 4$ attenuated $\mathrm{NF}-\kappa \mathrm{B}$, but less effectively than $\mathrm{Naf}^{\mathrm{FL}}$ or Naf1 $\alpha 2$.

Expression of the Nafl gene in AML patients. To investigate the expression of Naf1 variants on AML blasts, six clinical AML patients were selected. RT-PCR was performed with mRNA from PBMNCs from these six patients both at diagnosis and on remission after chemotherapy. The overall expression levels of the Nafl gene were not significantly altered between each paired sample from AML patients even in the presence of LOH (data not shown). Then the expression of Naf1 $\alpha$ variants on AML blasts was compared to PBMNCs from corresponding patients. Among PBMNCs obtained, AML blast percentage was $>74 \%$ at diagnosis and $<1 \%$ on their remission. Of the six AML patients, decreased expression of Naf1 $\alpha^{\mathrm{FL}}$ was observed in four $(67 \%)$; decreased expression of Naf1 $\alpha 3$ in five (83\%), while Naf1 $\alpha 2$ showed constant expression (Fig. 6). As to the Naf1 $\beta$ variants, all cases of blasts from the 5 AML patients expressed Naf1 $\beta^{\mathrm{FL}}$ but not in samples from healthy adults both in peripheral and bone-marrow cells, while expression of Naf1 B3 was ubiquitous (Fig. 6).

\section{Discussion}

Loss of chromosome 5, or del(5q), are recurring abnormalities in malignant myeloid diseases. Several groups of investigators have defined a commonly deleted segment on $5 \mathrm{q}$ predicted to contain a myeloid TSG on 5q31. We detected the highest frequency of LOH on 5q near the STS marker D5S2014, located on 5q32. We then hypothesized that the Nafl gene, which is located at $5 \mathrm{q} 32-5 \mathrm{q} 33.1$, might be the TSG for myeloid malignancies because Naf1 is reported to be an attenuator of TNF-induced NF- $\mathrm{KB}$ activation and of EGF/ERK2 nuclear signaling $(21,24)$.

It was previously reported that there are $\alpha$ and $\beta$ isoforms to $\mathrm{Naf}^{\mathrm{FL}}$ according to the variant splicing of exon 18 (17). We identified novel Nafl splice variants in the C-terminal region; i.e. deletion of exon 16 ( $\mathrm{Naf} 1 \alpha 2$ and B2), deletion of exon 16 with insertion (Naf1 $\alpha 3$ and B3) and deletion of exon 16 and exon 17 (Naf1 $\alpha 4)$. We found $\alpha$ and $\beta$ isoforms in each type of the novel isoforms except for Naf1 $\alpha 4$. Considering splice variants in the $\mathrm{N}$-terminal region, i.e. deletion exon 2 for both variants with exon $1 \mathrm{a}$ or exon $1 \mathrm{~b}$, the number of Naf1 splice variants would increase (Fig. 2A).

Most of the leukemia-lymphoma cell lines showed higher expression of $\mathrm{Naf}^{\mathrm{FL}}$, Naf1 $\alpha 3$ and $\mathrm{B} 3$ and lower expression of Naf1 $\alpha 4$ than PBMNCs from healthy adults. AML blasts also showed higher expression of $\mathrm{Naf}^{\mathrm{FL}}$, Naf1 $\alpha 3$ and $\mathrm{B3}$ than healthy adult PBMNCs.

To investigate whether there are functional differences among these Naf1 variants, a luciferase assay was performed. The NF-кB inhibitory effect of Naf1 $\alpha 2$ was similar to that of Naf1 $\alpha^{\mathrm{FL}}$. Naf1 $\alpha 4$ had less NF-кB inhibitory effect than Naf1 $\alpha 2$ or Naf1 $\alpha^{\mathrm{FL}}$. This result indicates that Naf1 $\alpha 2$, the main transcript of the Nafl gene in healthy adults, has almost equal $\mathrm{NF}-\kappa \mathrm{B}$ inhibitory effect to Naf1 $\alpha^{\mathrm{FL}}$. It seems that there is little functional difference between Naf1 $\alpha^{\mathrm{FL}}$ and Naf1 $\alpha 2$. However, considering the distinct expression pattern of Naf1 variants, there may be other functional differences not yet elucidated.

RT-PCR analysis revealed almost no expression of Naf1 $1^{\mathrm{FL}}$ in PBMNCs from healthy adults while its expression was higher in most of the leukemia-lymphoma and solid tumor cell lines (data not shown), as well as the AML clinical samples. Moreover, $\mathrm{Naf1} 1^{\mathrm{FL}}$ was the dominant transcript of the Nafl gene in some of the hematological cell lines (U937, K-562 and NALL-1) and solid tumor cell lines (HeLa, Saos2 and MKN45), while Naf1 $\alpha 2$ was the dominant transcript in PBMNCs from healthy adults, and PBMNCs from AML patients and in certain cell lines. Therefore, Naf1 $\alpha 2$, not $\mathrm{Naf} 1^{\mathrm{FL}}$, is the major product of the Nafl gene in healthy adults, unlike previous reports. We think this is a unique case where a gene's transcription variant with a spliced out exon presents the main product of the gene.

Although little difference in NF- $\mathrm{BB}$ inhibitory effect between Naf1 $\alpha^{\mathrm{FL}}$ and Naf1 $\alpha 2$ was observed, from the fact that almost no expression of $\mathrm{Naf} 1^{\mathrm{FL}}$ or Naf1 $\alpha 3$ in healthy adults in contrast to the clear expression of these transcripts in AML patients and immortalized cell lines, one can speculate that the expression of $\mathrm{Naf}^{\mathrm{FL}}$ and/or Naf1 $\alpha 3$ is related to cell immortalization and/or transformation. It is likely that some unknown functional differences exist among these variants other than the NF-кB inhibitory effect; for example, the attenuation potential of EGF/ERK nuclear signaling or the augmentation of cell surface CD4 expression, and these remain to be examined.

We next investigated whether there is any difference in the expression levels of the Naf1 variants in the same AML patients before and after chemotherapy. The expression levels of Naf1 $\alpha^{\mathrm{FL}}$ was decreased after therapy in four out of 
six $(67 \%)$ patients and Naf1 $\alpha 3$ were decreased in five out of six (83\%) patients (Fig. 6). Moreover, Naf1 $\alpha 3$ expression was higher in AML blasts than in normal PBMNCs suggesting the possibility that Naf1 $\alpha 3$ plays a role as an oncoprotein directly influencing the leukemogenesis or cell proliferation or inhibiting the tumor-suppressor effect of Naf1 in a dominantly negative manner, since the Naf1 $\alpha 3$ protein is devoid of the $\mathrm{C}$-terminal domains.

Finally, the relevance of LOH at $5 q 32$ to the observed splicing alterations in Naf1 mRNA is an important issue. The absence of a close relationship between these phenomena suggests that i) the true target gene for the allelic loss may not be the Nafl gene itself, ii) LOH perturbed normal splicing control of the Nafl gene, and iii) LOH affected the stability of some mRNA with unusual structure such as the Naf1variants. In relation to these possibilities, it was recently found that Naf1 also associates with TTF2/HuF2 protein that is implicated in pre-mRNA splicing (25). Furthermore, we recently demonstrated that prevalent $\mathrm{LOH}$ was accompanied by tumor-specific splicing alterations of the SMARCA6 gene in human primary lung cancer (26).

In summary, we have identified novel splice variants of Naf1. The deletion of exon 16 type variant (Naf1 $\alpha 2$ ), having equivalent activity as full-length $\mathrm{Naf1}\left(\mathrm{Naf} 1^{\mathrm{FL}}\right)$, was the dominant transcript in normal PBMNCs of healthy adults. Full-length $\mathrm{Naf} 1\left(\mathrm{Naf1}^{\mathrm{FL}}\right)$ and $\mathrm{Naf} 1 \alpha 3$ were only expressed in tumor/immortalized cell lines and PBMNCs of AML patients. Naf1 a3 terminates prematurely and lacks the Cterminal domain encoded by exons 16-18. In clinical AML patients, the expression of Naf1 $\alpha 3$ was much higher at diagnosis than on their remission after chemotherapy, suggesting a possible dominant negative effect of Naf1 $\alpha 3$.

\section{References}

1. Lowenberg B, Downing JR and Burnett A: Acute myeloid leukemia. N Engl J Med 341: 1051-1062, 1999.

2. Head DR: Revised classification of acute myeloid leukemia. Leukemia 10: 1826-1831, 1996.

3. He LZ, Tribioli C, Rivi R, Peruzzi D, Pelicci PG, Soares V, Cattoretti G and Pandolfi PP: Acute leukemia with promyelocytic features in PML/RARalpha transgenic mice. Proc Natl Acad Sci USA 94: 5302-5307, 1997.

4. Brown D, Kogan S, Lagasse E, Weissman I, Alcalay M, Pelicci PG, Atwater S and Bishop JM: A PMLRARalpha transgene initiates murine acute promyelocytic leukemia. Proc Natl Acad Sci USA 94: 2551-2556, 1997.

5. Grisolano JL, Wesselschmidt RL, Pelicci PG and Ley TJ: Altered myeloid development and acute leukemia in transgenic mice expressing PML-RAR alpha under control of cathepsin G regulatory sequences. Blood 89: 376-387, 1997.

6. Castilla LH, Garrett L, Adya N, Orlic D, Dutra A, Anderson S, Owens J, Eckhaus M, Bodine D and Liu PP: The fusion gene Cbfb-MYH11 blocks myeloid differentiation and predisposes mice to acute myelomonocytic leukaemia. Nat Genet 23: 144-146, 1999.

7. Rhoades KL, Hetherington CJ, Harakawa N, Yergeau DA, Zhou L, Liu LQ, Little MT, Tenen DG and Zhang DE: Analysis of the role of AML1-ETO in leukemogenesis, using an inducible transgenic mouse model. Blood 96: 2108-2115, 2000.

8. Tobal K, Saunders MJ, Grey MR and Yin JA: Persistence of RAR alpha-PML fusion mRNA detected by reverse transcriptase polymerase chain reaction in patients in long-term remission of acute promyelocytic leukaemia. Br J Haematol 90: 615-618, 1995.

9. Nucifora G, Larson RA and Rowley JD: Persistence of the 8;21 translocation in patients with acute myeloid leukemia type M2 in long-term remission. Blood 82: 712-715, 1993.
10. Barrett MT, Sanchez CA, Prevo LJ, Wong DJ, Galipeau PC, Paulson TG, Rabinovitch PS and Reid BJ: Evolution of neoplastic cell lineages in Barrett oesophagus. Nat Genet 22: 106-109, 1999.

11. Vogelstein B, Fearon ER, Hamilton SR, Kern SE, Preisinger AC, Leppert M, Nakamura Y, White R, Smits AM and Bos JL: Genetic alterations during colorectal-tumor development. N Engl J Med 319: 525-532, 1988.

12. Wong IH, Ng MH, Huang DP and Lee JC: Aberrant p15 promoter methylation in adult and childhood acute leukemias of nearly all morphologic subtypes: potential prognostic implications. Blood 95: 1942-1949, 2000.

13. Mizuno S, Chijiwa T, Okamura T, Akashi K, Fukumaki Y, Niho Y and Sasaki H: Expression of DNA methyltransferases DNMT1, $3 \mathrm{~A}$, and $3 \mathrm{~B}$ in normal hematopoiesis and in acute and chronic myelogenous leukemia. Blood 97: 1172-1179, 2001.

14. Casas S, Aventin A, Fuentes F, Vallespi T, Granada I, Carrio A, Martinez-Climent JA, Sole F, Teixido M, Bernues M, Duarte J, Hernandez JM, Brunet S, Coll MD, Sierra J: Genetic diagnosis by comparative genomic hybridization in adult de novo acute myelocytic leukemia. Cancer Genet Cytogenet 153: 16-25, 2004.

15. Le Beau MM, Albain KS, Larson RA, Vardiman JW, Davis EM, Blough RR, Golomb HM and Rowley JD: Clinical and cytogenetic correlations in 63 patients with therapy-related myelodysplastic syndromes and acute nonlymphocytic leukemia: further evidence for characteristic abnormalities of chromosomes no. 5 and 7. J Clin Oncol 4: 325-345, 1986.

16. Le Beau MM, Espinosa III R, Neuman WL, Stock W, Roulston D, Larson RA, Keinanen M and Westbrook CA: Cytogenetic and molecular delineation of the smallest commonly deleted region of chromosome 5 in malignant myeloid diseases. Proc Natl Acad Sci USA 90: 5484-5488, 1993.

17. Fukushi M, Dixon J, Kimura T, Tsurutani N, Dixon MJ and Yamamoto N: Identification and cloning of a novel cellular protein Naf1, Nef-associated factor 1, that increases cell surface CD4 expression. FEBS Lett 442: 83-88, 1999.

18. Heyninck K, De Valck D, Vanden Berghe W, Van Criekinge W, Contreras R, Fiers W, Haegeman G and Beyaert R: The zinc finger protein A20 inhibits TNF-induced NF-kappaB-dependent gene expression by interfering with an RIP- or TRAF2-mediated transactivation signal and directly binds to a novel NF-kappaBinhibiting protein ABIN. J Cell Biol 145: 1471-1482, 1999.

19. Cooper JT, Stroka DM, Brostjan C, Palmetshofer A, Bach FH and Ferran C: A20 blocks endothelial cell activation through a NF-kappaB-dependent mechanism. J Biol Chem 271: 1806818073,1996

20. Lee EG, Boone DL, Chai S, Libby SL, Chien M, Lodolce JP and Ma A: Failure to regulate TNF-induced NF-kappaB and cell death responses in A20-deficient mice. Science 289: 2350-2354, 2000.

21. Zhang S, Fukushi M, Hashimoto S, Gao C, Huang L, Fukuyo Y, Nakajima T, Amagasa T, Enomoto S, Koike K, Miura O, Yamamoto $\mathrm{N}$ and Tsuchida N: A new ERK2 binding protein, Naf1, attenuates the EGF/ERK2 nuclear signaling. Biochem Biophys Res Commun 297: 17-23, 2002.

22. Gupta K, Ott D, Hope TJ, Siliciano RF and Boeke JD: A human nuclear shuttling protein that interacts with human immunodeficiency virus type 1 matrix is packaged into virions. J Virol 74: 11811-11824, 2000

23. Favre M, Butticaz C, Stevenson B, Jongeneel CV and Telenti A: High frequency of alternative splicing of human genes participating in the HIV-1 life cycle: a model using TSG101, betaTrCP, PPIA, INI1, NAF1, and PML. J Acquir Immune Defic Syndr 34: 127-133, 2003.

24. Heyninck K, Kreike MM and Beyaert R: Structure-function analysis of the A20-binding inhibitor of NF-kappa B activation, ABIN-1. FEBS Lett 536: 135-140, 2003.

25. Leonard D, Ajuh P, Lamond AI and Legerski RJ: hLodestar/HuF2 interacts with CDC5L and is involved in premRNA splicing. Biochem Biophys Res Commun 308: 793-801, 2003.

26. Yano M, Ouchida M, Shigematsu H, Tanaka N, Ichimura K, Kobayashi K, Inaki Y, Toyooka S, Tsukuda K, Shimizu N and Shimizu K: Tumor-specific exon creation of the HELLS/ SMARCA6 gene in non-small cell lung cancer. Int J Cancer 112: 8-13, 2004 . 